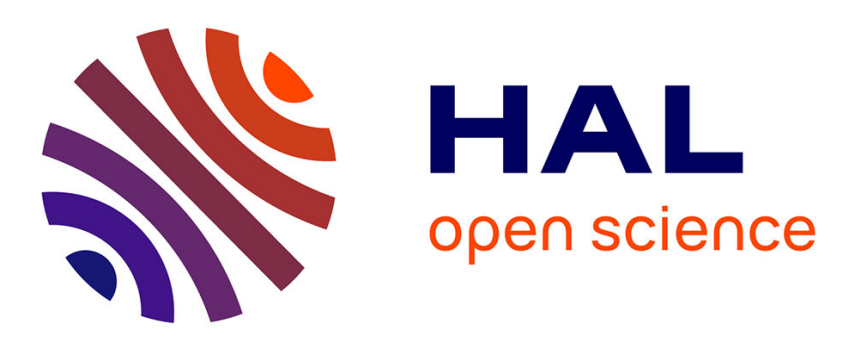

\title{
Une approche phénoménologique du récit de soi : Michel Foucault et la généalogie historique des signes Jacques Guilhaumou
}

\section{To cite this version:}

Jacques Guilhaumou. Une approche phénoménologique du récit de soi : Michel Foucault et la généalogie historique des signes. Semen - Revue de sémio-linguistique des textes et discours, 2014, 37, pp.133150. 10.4000/semen.10221 . halshs-00995355

\section{HAL Id: halshs-00995355 https://shs.hal.science/halshs-00995355}

Submitted on 25 Mar 2020

HAL is a multi-disciplinary open access archive for the deposit and dissemination of scientific research documents, whether they are published or not. The documents may come from teaching and research institutions in France or abroad, or from public or private research centers.
L'archive ouverte pluridisciplinaire HAL, est destinée au dépôt et à la diffusion de documents scientifiques de niveau recherche, publiés ou non, émanant des établissements d'enseignement et de recherche français ou étrangers, des laboratoires publics ou privés. 


\title{
Une approche phénoménologique du récit de soi. Michel Foucault et la généalogie historique des signes
}

\author{
Jacques GUILHAUMOU,
}

in Semen $\mathrm{N}^{\circ} 37,2014$, Approches discursives des récits de soi, p. 133-150.

$\mathrm{Au}$ cours de sa formation intellectuelle, Michel Foucault a été marqué conjointement par la lecture de Nietzsche et celle de la phénoménologie, Heidegger et Husserl principalement. De Nietzsche à Husserl (Vioulac, 2005), il conçoit un travail généalogique, au plus près de l'histoire « réelle » liée à l'expérience du vécu, qui permet de se centrer sur la vie du sujet, tout en se mettant à distance d'une approche strictement analytique de la rationalité. Il fait ainsi sien le mot d'ordre de "retour réflexif vers soimême » de la phénoménologie, en particulier à la lecture du «testament » de Husserl, son ouvrage inédit de son vivant sur La crise des sciences européennes et la phénoménologie transcendantale (1976). Mais dans le même temps, il est marqué par la lecture de Raymond Roussel, et son dernier ouvrage, Comment j'ai écrit certains de mes livres (1935). Il situe cet auteur dans le sillage de Nietzche en concevant une rupture menée de front par ces deux auteurs en matière d' « œuvre de langage », située au plus près de la folie, un de ses thèmes majeurs de recherche (1972) en ses débuts.

L'objectif de la présente étude est de circonscrire un « récit de soi » chez Michel Foucault à partir des bornes épistémologiques posées par Nietzsche et Roussel, en matière de généalogie historique et d'archéologie du discours. Je m'en tiens au corpus de ses Dits et écrits rassemblés en quatre volumes (1994) ${ }^{1}$. Ce choix de corpus correspond au fait qu'une partie de ses textes sont issus d'entretiens. Relevant de l'oralité pour une part, ils incitent Michel Foucault à se positionner, dans l'échange, sur ce qu'il en est de lui-même sous le regard des autres. Il précise par ailleurs : «Je crois qu'il vaut mieux essayer de concevoir que, au fond, quelqu'un qui est écrivain ne fait pas simplement son œuvre dans ses livres, dans ce qu'il publie, et que son œuvre principale, c'est finalement lui-même écrivant ses livres » (IV, 607). Soucieux de son rapport à la vie, de son vécu propre, Foucault entre

\footnotetext{
${ }^{1}$ Par la suite, les références aux textes de Michel Foucault sont indiquées entre parenthèses dans le texte avec le numéro du volume et la page.
} 
dans un « récit de soi » à partir de la question récurrente : «Que pourrai-je être pour moi-même ? » comme l'a souligné Judith Butler $(2007,116)$.

\section{L'ordre des signes confronté aux significations immanentes de l'expérience vécue}

Dès le départ, Michel Foucault considère que le sujet, saisi dans son contexte historique, sa vie même, est seul source du sens, qu'il est donc sujet du discours en prise sur son vécu dans l'histoire « réelle », à l'encontre de toute approche purement objective. À ce titre, le moi fait retour sur le monde propre, et par là même son propre monde, dans l'immanence de la vie. En dialogue permanent avec Gilles Deleuze, Foucault conçoit ainsi ce monde propre selon une logique immanente des signes inscrite à l'horizon d'un devenir. De manière plus historique, il s'efforce d'appréhender la transvaluation de l'esprit et de ses valeurs dans le champ d'expérimentation du vécu discursif. C'est à ce titre qu'il fait référence de manière constante à un continuum de l'être à la perception du réel, de l'essence à l'existence, sur la base d'une ontologie des signes en rapport à l'immanence de l'événement.

Se situant entre un objet de recherche, l'investigation archéologique de la théorie des signes en appui sur l'ordre empirique et sa constitution, et une approche généalogique d'ordre historique, Foucault considère qu'après un XIXème siècle où «l'homme a existé là où le discours s'est tu » (I, 501), tout change lorsque l'homme, de Nietzsche à Husserl, meurt des signes qui sont en lui pour devenir l'être même des signes, l'être du langage. Il se rapproche ainsi de Saussure et des linguistes néo-saussuriens du XXème siècle en définissant les contours d'une sémiotique de la transversalité et de la transvaluation, c'est-à-dire en considérant l'unicité du signe sur la base de la bifacialité signifiant/signifié et de la différencialité (la valeur purement différentielle du signe).

Il nous faut ici prendre en compte les travaux récents des historiens sur le « récit de soi », tant du côté de l'engagement de soi que de la souffrance de soi (Bardet, Arnoul \& Ruggiu, 2010 ; Luciani \& Pietri, 2012 ; Atlan \& Luciani, 2014). Les historiens nous incitent, de concert avec les sociologues et les anthropologues, à considérer les réalisations historiques des processus de subjectivation en nous interpellant sur les modes d'expression de soi au plus près des capacités réflexives de l'acteur/auteur. Ces interrogations concernent aussi les figures de philosophes, mais surtout de la période moderne (Ribard, 2003).Le choix de Foucault porte sur une philosophie de tradition phénoménologique. Pourquoi ce choix ? Revenons à la lecture que fait Husserl de Descartes dans ses Méditations cartésiennes (1992). De manière paradoxale, il retient de Descartes, dont il est fort critique par ailleurs, la nécessité, pour le philosophe, de «se replier sur soi-même, et au 
dedans de soi » $(1992,18)$ par une prise de distance radicale avec les sciences existantes. Il s'agit de mettre l'accent sur « la présence vivante du moi à lui-même ». Alors l'expression «Je suis » vise «quelque chose, et qu'il porte en lui-même, en tant que visé (en tant qu'objet d'une intention), son cogitatum respectif» $(1992,65)$, et que je traduis (2006) par l'expression «quelque chose existe». À ce positionnement phénoménologique sur le «Je suis », que Michel Foucault fait sien, s'ajoute le «Je dis » qu'il retient de la tradition anglo-saxonne en philosophie du langage, en particulier Wittgenstein, Austin, Strawson et Searle, et que l'on peut traduire par la formule «quelqu'un parle». C'est dans ce cadre épistémologique - quelque chose existe et quelqu'un parle - que se situe l'héritage qu'il nous laisse en analyse de discours.

Peut-on alors considérer que Michel Foucault ouvre ainsi la voie à un « récit de soi » dans son œuvre même ? Peut-on montrer que sa volonté de considérer prioritairement le monde de la vie et des signes selon une logique discursive immanente relève d'un retour sur moi-même? Qu'en est-il de son «récit de soi » lorsqu'il situe en permanence ses questionnements à l'horizon d'une théorie du sujet pensé dans le monde des signes ? Même s'il ne publie pas un texte ayant le statut explicite de « récit de soi », n’opère-t-il pas un retour réflexif sur lui-même, par toutes sortes de médiations discursives?

En 1968 dans un entretien (I, 651), il précise qu'il a commencé à écrire de façon continue lors de son séjour en Suède, où, face au mutisme des Suédois, il s'est mis au «bavardage inépuisable »-ce sont bien ses termes, en lien avec ses lectures continues qui ne cessent d'enrichir sa très grande culture. Il fournirait ainsi une première clé de son moi-même. Mais, en même temps, il précise que "l'œuvre comporte toujours pour ainsi dire la mort de l'auteur lui-même. On n'écrit que pour autant disparaître. L'œuvre existe en quelque sorte par elle-même, comme l'écoulement » (I, 660). Il ne prétend donc à la construction d'une œuvre que pour autant disparaître derrière la multitude des signes qu'elle produit.

\section{Les années 1960 : Le récit de soi à l'horizon de l'expressivité des signes}

\subsection{L'apport de la littérature : du secret à l'expressivité du langage}

La première expérimentation historique d'ampleur à laquelle Foucault se confronte est celle de la folie. En 1964, il résume son approche de la folie dans une intervention significativement intitulée «La folie, l'absence d'œuvre » (I, 412). D'un point de vue généalogique, il considère que l'homme classique, «l'être du départ, du retour et du temps [...] l'étranger à 
soi qui redevient familier » $(\mathrm{I}, 414)$ a su mettre à distance cette figure de soimême, le fou. Mais il a été recouvert ensuite par le contrôle technique de la folie. Reste que «le mouvement qui referme sur soi l'expérience de la folie » (I, 415), se déplace vers la littérature. Ainsi Foucault définit la littérature contemporaine dans les termes suivants : « la littérature (et cela depuis Mallarmé sans doute) est en train peu à peu de devenir à son tour un langage dont la parole énonce, en même temps que ce qu'elle dit et dans le même mouvement, la langue qui la rend déchiffrable comme parole » (I, 418). Deux écrivains vont alors retenir son attention, Raymond Roussel et Jean-Jacques Rousseau.

La présence constante de Raymond Roussel, auquel il consacre un livre peu connu de son vivant (Raymond Roussel, Gallimard, 1963), marque bien la part de l'ombre en moi-même. Raymond Roussel fait partie de ses auteurs qui marquent fortement plusieurs générations d'écrivains et de lecteurs, tout en ayant connu des échecs commerciaux dans la publication de leurs écrits. Leur spécificité tient en grande part à des exercices linguistiques à la limite de la thérapie, sans s'y réduire ${ }^{2}$. Tout d'abord le surréalistes le revendiquent comme l'un de leurs maîtres: André Breton le premier, au même titre qu'un autre inconnu du public, Jean-Pierre Brisset, puis Michel Leiris qui me la fait connaître avant même Foucault. Actuellement, l'intérêt des lecteurs et des critiques de Raymond Roussel porte sur sa capacité à scénariser, d'un ouvrage à l'autre, la vie dans les moindres détails, ce qu'on peut qualifier de travail aux limites du langage. Il s'agit donc objectivement d'un écrivain de l'ombre, mais subjectivement pris dans la complexité même de la conscience du secret de sa vie.

C'est pourquoi, avec Foucault, tout se joue autour du dernier livre de Roussel, Comment j'ai écrit certains de mes livres ${ }^{3}$, qu'il analyse longuement dans son ouvrage bien sûr, mais aussi dans la prépublication quelque peu modifiée du chapitre I en 1962. Il y revient dans sa dernière année d'écriture (1984) sous un titre particulièrement significatif d'un aspect central de son récit de soi, "Archéologie d'une passion» (IV, 599). Il précise alors que Roussel est pour lui un adepte majeur du «jeu de langage » dont Wittgenstein se fera le théoricien dans la mesure où il met au centre de sa réflexion « le fait que nous vivons dans un monde dans lequel il y a eu des choses dites » (IV, 602). Ainsi, tout est, avec Roussel, affaire de visibilité/invisibilité dans son rapport à soi, par le fait même de la médiation

\footnotetext{
${ }^{2}$ L'écrivain et critique littéraire Bruno Clément a fait de cette spécificité linguistique un objet central de ses travaux. Voir en particulier L'ouvre sans qualités. Rhétorique de Samuel Beckett, Paris, Seuil, 1994.

${ }^{3}$ Editions Alphonse Lemerre, 1935.
} 
du langage. Il en ressort un langage labyrinthique que Foucault lui-même explore diversement à travers les variantes de son chapitre I. Mais dans quel sens? Je m'en tiens à deux exemples particulièrement significatifs selon moi.

Foucault rajoute dans le livre à la première version (I, 1962, 206), une citation de Roussel : «Et je me réfugie faute de mieux dans l'espoir que j'aurai peut-être un peu d'épanouissement posthume à l'endroit de mes livres» (1963, 9), ainsi que l'analyse suivante: «on y trouve des renseignements, point de confidence, et pourtant quelque chose est confié, absolument » $(1963,8)$. Quelque chose existe bien chez Roussel, de manière ontologique et confident, moi-même donc.

À l'inverse, on peut repérer la réécriture suivante, plutôt complexe, en comparant l'ouvrage et le pré-chapitre. Foucault écrit en premier lieu : «l'impossibilité ici de décider lie tout discours sur Roussel non seulement au risque commun de se tromper mais à celui, plus raffiné, de l'être - et par la conscience même du secret, toujours tenté de le refermer sur lui-même et d'abandonner l'œuvre à une nuit facile, toute contraire à l'énigme du jour qui la traverse » (I, 1962, 207). Puis, dans son livre, il réduit ce passage de la façon suivante: "l'impossibilité ici de décider lie tout discours sur Roussel non seulement au risque commun de se tromper mais à celui, plus raffiné, de l'être. Et d'être trompé moins par un secret que par la conscience qu'il y a un secret» $(1963,10)$. L'expression initiale de la «conscience même du secret », qui désigne un secret se renfermant sur luimême par un jeu de langage, marque le risque d'abandonner le langage à lui-même, au détriment de l'expérience propre à moi-même, de mon œuvre. La réécriture simplifie alors cette approche de moi-même, en gommant une positivité, la dimension intentionnelle du langage qui confère ou non une réalité à l'œuvre, et en s'en tenant à une négativité, la réduction à la conscience, source d'illusion.

C'est là, me semble-t-il, que se situe la difficulté à se retrouver dans les écrits de Foucault en matière de « récit de soi ». Par un bavardage incessant, selon son propre dire, il exhibe un langage à la fois limpide et labyrinthique, pour mieux en garder le secret en ce qui concerne lui-même, tout en suivant le critère phénoménologique de l'expérience vécue. En effet, il s'en tient, dans l'ordre de la généalogie historique, aux « significations immanentes au vécu » - voir son interview de 1967 sur sa formation phénoménologique (I, 601) -, donc à ce que je dis de l'expérience des événements d'un point de vue historique, en parcourant un trajet du thème de la folie à celui du «gouvernement de soi » $(2008,5)$. 
L'autre auteur de référence est Rousseau. En 1962, Foucault a charge d'introduire une édition de Rousseau juge de Jean-Jacques. Dialogues (I, 172, svtes). Partant de l'idée qu'il s'agit d' «anti-Confessions », il s'efforce de marquer la différence entre les Dialogues et les Confessions du point de vue de leur rapport aux signes. Selon lui, le langage des Confessions est le plus souvent linéaire. Soucieux de faire connaître au public «tous les recoins de sa vie», Rousseau se centre sur son «Moi seul » à l'aide d'une expression continue, et fidèle au cours du temps, marquant ainsi son «inséparable proximité à soi-même et absolue différence avec les autres » (I, 176). On se doute bien que nous ne sommes pas là dans la démarche qui intéresse alors Foucault très peu adepte de cette transparence à soi-même. À l'inverse, les Dialogues retiennent beaucoup plus son attention. Foucault y situe de suite « un sujet qui parle », « un sujet dissocié, superposé à lui-même, lacunaire » qui n'est présent que dans son inachèvement même. Ici se situe la vérité de moi-même. Les Dialogues sont ainsi une confrontation entre trois personnages : un "Rousseau réel », un français anonyme qui lui a volé son nom, et une tierce personne, le JeanJacques hautain dont l'image a été construite par ses détracteurs. Rousseau lui-même dégage de cette confrontation un quatrième personnage, le JeanJacques écrivant ses livres dissocié d'un Jean-Jacques faussaire, donc criminel auprès de l'opinion. Foucault se sent-il en affinité avec cet ultime Rousseau ? Et dans quel sens ? Il ajoute alors que, dans les Dialogues, «il s'agit, dans un seul mouvement de pensée, de fonder l'inexistence et de justifier l'existence » $(\mathrm{I}, 179)$ - du négatif et du positif donc - ce qui lui permet d'affirmer conjointement : " les Dialogues s'efforcent de faire naître un langage à l'intérieur d'un espace où tout se tait » (180); «et dans ce monde qui s'enchante de la réalité elle-même, les signes sont dès l'origine pleins de ce qu'ils veulent dire. Ils ne forment un langage que dans la mesure où ils détiennent une immédiate valeur expressive » (I, 185-186). Précisons alors ce qu'il en est d'une telle approche de l'expressivité des signes, véritable «moment linguistique » sous l'égide de la Grammaire de Port-Royal et de Saussure.

\subsection{Le moment linguistique : remontons de Saussure à la Grammaire de Port-Royal 1967-1969}

De Saussure, la génération des années 1970 retient un mode spécifique de perception et d'appréhension du signe et de l'énoncé, sur la base du Cours de linguistique générale (1916) et dont la publication récente des manuscrits (Saussure, 2002) a confirmé les analyses majeures, au-delà de la simple lecture structuraliste. Si le signe existe hors de l'historicité des formes, par association faite par l'esprit avec une idée, appréhendée dans son 
déploiement historique, il est tout autant dénué de signification a priori parce qu'il n'est pas délimité en soi. Il n'y a donc pas d'êtres linguistiques donnés en soi, mais seulement des différences entre les signes issus de la combinaison de la forme et du sens perçu. On ne peut donc isoler les termes puisqu'ils n'ont de réalité linguistique que sous forme de rapports.

C'est pourquoi le discursif et le social ne sont pas des réalités distinctes : ils sont deux manières de caractériser la même chose, le système de signes constitutif de la langue. Le signe existe à la fois dans notre esprit et par le lien social du fait même de la constitution de la langue. Quant au fait social de la langue, il est attesté dans la combinaison sociale de la diversité mécanique des idées et de la diversité organique des signes.

Foucault perçoit alors la rupture saussurienne comme le passage d'une philologie historique qui se donne pour vocation l'étude de la totalité de l'évolution d'une langue à une méthodologie résolument partielle (I, 610) par souci de mettre en valeur des phénomènes linguistiques spécifiques. La langue est alors considérée comme une « institution sociale » (Saussure), en tant qu'elle est « un système de signes » comparable avec d'autres systèmes sémiotiques, tout en étant le plus important ${ }^{4}$. A une telle base méthodologique prise dans un horizon social, il s'agit alors associer «une forme d'activité » (I, 612) qui ouvre à une nouvelle découverte scientifique.

C'est là où Foucault revendique l'invention du discours comme objet d'histoire sur la base méthodologique d'une archéologie du discours et de l'invention généalogique de l'activité humaine d'un sujet parlant. On peut donc considérer que la manière dont il parle des séquences linguistiques au sein des configurations discursives relève d'une telle approche saussurienne du signe.

Remontons alors, avec Foucault, de la linguistique contemporaine à la Grammaire de Port-Royal (I, 732 svtes). En premier lieu l'analyse de la langue dépend d'une théorie du signe, et non l'inverse. Il en ressort que la langue est fondée sur un ordre des raisons associé aux principes liés à ce que les hommes veulent dire en général. La Logique comme art de penser renvoie aux actions de l'esprit, et non à un art de bien penser. Elle est «l'art de penser s'éclairant de lui-même » (I, 741). Elle correspond à un art de parler, rien de plus, c'est à dire à une tâche purement réflexive. Il en ressort une théorie du signe placée au cœur de la réflexion sur l'idée. La rencontre entre la linguistique historique et la linguistique contemporaine se fait ici sur la nouvelle manière d'aborder la théorie du signe, et son corollaire, la façon de concevoir le travail de l'esprit propre au sujet parlant. Quant au lien

${ }^{4}$ Le livre de Claudine Normand, Saussure, Paris, Les Belles Lettres, 2000 restitue bien une telle lecture du Cours de linguistique générale dans la génération des années 1970-1980. 
du langage à la vie, si souvent évoqué par les linguistes, il renvoie à un continuum de variation d'un individu à l'autre, par le fait même de l'existence de son être au monde. Le sujet parlant comme individu est porteur de ses conditions internes d'existence, sans référence à une individualité toute faite. C'est là également, où s'effectue, selon Foucault, le lien entre Nietzsche et le langage, selon la définition suivante dans un entretien de 1967, récemment publié, en complément des Écrits (Cahiers de l'Herne, 2010, 109) : "Nous découvrons maintenant que Nietzsche, lui aussi, a mis en question le langage. Et non seulement pour retrouver, en bon philologue, la forme rigoureuse et le sens exact de ce qui a été écrit ; non seulement pour mettre au jour, en bon exégèse, les significations cachées ; mais pour interroger notre existence et l'être même du monde, à partir de ce que nous disons ; pour savoir qui parle dans tout ce qui se dit». Qui parle lorsque «je dis» dans le courant de mon vécu, telle est la spécificité linguistique d'une généalogie nietzschéenne dont Foucault n'a eu de cesse de préciser les contours.

\section{Les années 1970 : de l'autre côté du discours.}

J'en viens à son très célèbre texte de 1971 sur «Nietzsche, la généalogie, l'histoire» (II, 136) qui donne tous les éléments pour comprendre ce qu'il entend par "généalogie historique ", même s'il y revient, là encore, au cours de sa dernière année de publication, en 1984, pour en proposer une extension dans un texte intitulé « À propos de la généalogie de l'éthique : un aperçu d'un travail en cours » (IV, 609). Mais c'est aussi une période, sous l'angle de la présentation de soi (Amossy, 2010), où ses engagements nombreux, tout particulièrement à propos des prisons et autres institutions d'enfermement, le portent vers des enquêtes à portée militante. Ainsi en est-il de ses activités au sein du Groupe d'information sur les prisons, du Groupe d'information santé, du Groupe d'information sur les asiles, et enfin de celui pour le soutien aux travailleurs immigrés.

\subsection{Les contours d'une généalogie historique}

Dès 1967, dans un entretien sur le thème «Qui êtes-vous ? », Foucault insiste sur le fait que Nietzche opère " un travail de diagnostic » à propos de la nature de mon «aujourd'hui », au moment même où Husserl, dans son dernier texte sur La crise des sciences européennes tente aussi une généalogie de son contemporain à propos de l'idée d'Europe. Cependant, dans le texte-phare de 1971, Foucault part du fait que la généalogie demande de la patience, par la minutie du savoir qu'elle met en place. Elle est donc en lien avec une véritable discipline intellectuelle et méticuleuse, 
l'archéologie du savoir, qui n'est pas à proprement parler une méthode, mais un objet, c'est-à-dire un espace d'émergence d'objets possibles sous forme de règles ( «L'analyse archéologique est l'analyse [...] de la manière dont les objets sont constitués, les sujets se posent et les concepts se forment », II, 162). Qui plus est, la démarche généalogique n'est pas une recherche de l'origine au sens usuel. Elle répond en effet à trois critères : «la provenance, l'émergence et l'invention». Repérer une provenance, c'est « maintenir ce qui s'est passé dans la dispersion qui lui est propre » (II, 141), au sein de l'événement donc. Situer une émergence, c'est trouver un point de surgissement par le fait même de l'existence d' " une loi singulière d'une apparition» (II, 143), ce qui suppose un certain état des forces en présence dans l'événement. Quant à l'inventeur, il se tourne, en fin de parcours, vers «l'histoire effective», celle des discontinuités au sein d'une myriade d'événements jusqu'aux plus minuscules que l'on ne voit qu'en regardant ce qui se passe en bas.

\subsection{Parler à la suite de la parole de l'autre}

Cependant son rapport à la parole, en particulier savante, se complexifie. Au cours de sa leçon inaugurale au Collège de France (1971), il dit : «Plutôt que de prendre la parole [...], j'aurais aimé m'apercevoir qu'au moment de parler une voix sans nom me précédait depuis longtemps ; il m'aurait suffi alors d'enchaîner, de poursuivre la phrase, de me loger, sans qu'on y prenne bien garde, dans ses interstices, comme si elle m'avait fait signe en se tenant, un instant, en suspens » $(1971,7)$. Il se veut donc hors du discours dominant certes, mais surtout de l'autre côté du discours. Il s'agit non pas d'entrer de soi-même dans le discours, à l'injonction de l'institution, mais de situer le discours autour de soi comme «une transparence ouverte » dans l'horizon d'attente de la vérité, ce qu'il appelle «l'inquiétude du discours», et en confrontation avec le vécu de son «existence transitoire ». Il prend ainsi une distance radicale vis-à-vis du principe de la biographie intellectuelle (II. 12), jugée trop transparente au nom propre de l'auteur.

Par ailleurs, lorsque Jacques Chancel le convoque en 1975 à son émission «Radioscopie » pour «une radioscopie de lui-même» (II, 783 svtes), il précise qu'il a certes « barboté » dans le savoir depuis son enfance, mais qu'il toujours eu le souci d'érotiser le savoir, d'y prendre plaisir - il a la frénésie du savoir - jusqu'à vouloir susciter un plaisir physique chez son lecteur. La même année, interpellé par la question « Pourquoi Foucault est-il de gauche ? » par un Maurice Clavel qui vient de changer de camp, il répond là très simplement que c'est la gauche qui a pris en considération les questions qu'il a fait émerger sur la scène politico-intellectuelle avec l'aide 
des Groupe d'information sur les prisons. Il définit ensuite sa position critique de la façon suivante: «Je ne veux pas faire une critique qui empêche les autres de parler, exercer en mon nom un terrorisme de la pureté et de la vérité. Je ne veux pas non plus parler au nom des autres et prétendre dire mieux ce qu'ils ont à dire. Ma critique a pour objectif de permettre aux autres de parler, sans mettre de limites au droit qu'ils ont de parler » (II, 816).

Et là encore, alors que nous pouvons constater la montée en puissance de sa quête de l'historicité des régimes de vérité, il déroute celui qui l'écoute, en rétorquant, dans une conversation de la fin des années 70 , à son interlocuteur qui le désigne comme «non seulement critique, vous êtes, en outre un rebelle » : « Mais pas un rebelle actif. Je n'ai jamais défilé avec les étudiants et les travailleurs, comme le fait Sartre. Je crois que la meilleure forme de protestation est le silence, la totale abstention » (III, 670). De nouveau, et toujours, le silence donc, de l'auteur face à l'œuvre, et de ce qu'elle dit d'une forme particulière de « récit de soi ». Michel Foucault avait déjà précisé, très tôt (1968), que l'unité de l'œuvre ne se trouve que dans sa «fonction d'expression » (I, 703) par le fait que l'œuvre est l'expression d'une opération interprétative, disons de nature à la fois ontologique (quelque chose existe) et langagière (quelqu'un parle).

Ai-je vraiment avancé dans ce qu'il en est du « récit de soi » chez Foucault? D'un côté, la complémentarité de l'archéologie et de la généalogie se précise de plus en plus au fil des années, sans doute par le fait qu'elle correspond à la construction d'une œuvre. D'un autre côté, la présence du «récit de soi » est toujours plus dans l'ombre, au point de me renvoyer au silence de Foucault lui-même au sein même d'un "bavardage » en extension permanente avec le nombre toujours grandissant de pages qu'il écrit. Les Dits et écrits de la seconde moitié des années 1970 peuvent-ils alors nous permettre de sortir de ce paradoxe situant mon interrogation sur ce qu'il en est du nom de Foucault sous l'auto-désignation moi-même dans un espace réflexif de plus en plus incertain?

\subsection{La vérité de l'histoire}

Rappelant les domaines dans lesquels «j'ai essayé de faire une généalogie », et d'abord la psychiatrie, il précise que "l'histoire que j'ai faite» tente «la gageure» de tenir «un discours vrai» au titre du questionnement suivant: "Comment la vérité de l'histoire peut avoir politiquement un effet ?» (III, 29). Il s'agit certes bien là d'un "récit de soi » ponctué en «je », au nom d'un engagement très prégnant dans ses activités des années 1970, nous l'avons vu. Mais Foucault passe très vite sur 
le registre de la généralité en accordant à ce qu'il appelle désormais «le régime de vérité » une place centrale dans sa réflexion :

La vérité est de ce monde ; elle y est produite grâce à de multiples contraintes. Et elle y détient des effets réglés de pouvoir. Chaque société a son régime de vérité, sa 'politique générale' de la vérité ; c'est-à-dire les types de discours qu'elle accueille et fait fonctionner comme vrais; les mécanismes et les instances qui permettent de distinguer les énoncés vrais ou faux, la manière dont on sanctionne les uns et les autres; les techniques et les procédures qui sont valorisées pour l'obtention de la vérité ; le statut de ceux qui ont la charge de dire ce qui fonctionne comme vrai (III, 112).

Ainsi l'archéologie du savoir transvalue, par le fait de trouver enfin ses effets pratiques, sa définition dans l'étude des régimes de vérité. Ici un régime de vérité renvoie à « un ensemble de procédures réglées pour la production, la loi, la répartition, la mise en circulation, et le fonctionnement des énoncés » (III, 160). Affirmant qu'il a beaucoup écrit « sur la notion de soi et les techniques de soi » (IV, 610), Foucault se donne in fine l'objectif de faire aboutir son approche généalogique, complémentaire de ses enquêtes archéologiques, sur la base "d'une ontologie historique de nous-mêmes dans nos rapports à la vérité » (IV, 618). Il veut en effet construire sur une telle base non seulement «une ontologie historique de nous-mêmes dans nos rapports à un champ de pouvoir », déjà bien avancée dans ses dernières publications et ses cours, mais surtout « une ontologie historique de nos rapports à la morale », à venir donc (IV, 618).

La quête des « régimes de vérité » à travers l'histoire est bien l'objectif avéré, sans cesse réitéré, de Michel Foucault. Essayons de conclure sur la manière dont s'ouvre ainsi un nouvel espace de visibilité sur sa propre vérité, sur son moi-même. Un moi-même qui lui est propre, mais qu'il est difficile de circonscrire dans une œuvre qui ne revendique pas une conscience pleine et entière de son auteur, - illusion à ses yeux -, mais qui aspire à une présence authentique au monde sous le regard de ses lecteurs.

\section{Conclusion. Un Moi actuel en devenir}

Les textes de Michel Foucault publiés en 1984, sa dernière année de publication, peuvent être appréhendés, en première lecture, selon l'hypothèse suivante. Au centre de sa réflexion «ultime » se trouvent deux textes sur Qu'est-ce que les Lumières ? commentant des écrits de Kant sur les Lumières et la Révolution française comme événement (IV, 562-577 et 679-687), et sur la base de son cours du 5 janvier 1983 (2008, 9-21). Je peux alors considérer que ces écrits sont encadrés par deux autres textes, un 
premier texte intitulé Archéologie d'une passion qui revient, une ultime fois et sous forme d'entretien, sur l'apport central de Raymond Roussel pour comprendre la réflexivité de son œuvre (IV, 599-608); un second entretien sur La généalogie de l'éthique qui étend sa démarche généalogique à un projet de construction d' " une ontologie historique de nos rapports à la morale » (IV, 609-630). À propos de Roussel, il rappelle sa dette à son égard en matière de « jeu de langage » qui renvoie au fait « que nous vivons dans un monde qui est tout tramé, tout entrelacé de discours, c'est-à-dire d'énoncés qui ont été effectivement prononcés » (IV, 602). Roussel est bien là : il a toujours été une introduction à l'archéologie du discours. Certes archéologie et généalogie n'ont pas toujours été concomitantes dans le trajet de Foucault (Paltrinieri, 2012), mais leur complémentarité grandissante permet d'entrer de plein pied dans «une ontologie historique de nousmêmes ».

Cependant, interpellé par le questionnement généalogique et pris dans le labyrinthe des énoncés, le philosophe généalogiste et archéologue dispose-t-il encore d'un espace pour un regard sur lui-même ? La place occupée par Kant et les Lumières entre la généalogie et l'archéologie, les deux piliers de la démarche foucaldienne, ouvre-t-elle une interrogation sur un « je » équivalent à « moi-même »?

La question posée, à partir de Kant, est celle de l'événement présent, de sa singularité différentielle. Au-delà d'un usage de la raison qui est « affaire du sujet-lui-même en tant qu'individu » (IV, 566), il s'agit de circonscrire l'usage public de la raison, qui permet à tout citoyen de trouver une issue à son état de minorité face à l'autorité. Confronté à la question «Qu'est-ce qui se passe aujourd'hui ? » $(2008,13)$, ce sujet-citoyen est redevable d'une manière de penser, de sentir, une éthique en quelque sorte. Il est responsable de l'attitude qu'il adopte face à l'événement, et qui lui permet de se constituer soi-même comme sujet autonome.

Foucault se donne alors comme programme de travail «l'analyse de nous-mêmes comme sujets autonomes », qui se veut avant tout réflexive, dans la mesure où il s'agit d' " une critique et une création permanente de nous-mêmes dans notre autonomie » (IV, 573). Et de définir une fois de plus l'encadrement de cette critique : «elle est généalogique dans sa finalité et archéologique dans sa méthode ». "Travail indéfini de la liberté... aux limites de nous-mêmes » (IV, 574), une telle approche critique nécessite une attitude expérimentale. Revenant à l'expression en « je », Foucault se situe alors lui-même au sein d'une épreuve historico-pratique des limites que je peux franchir à tout moment. Et de conclure «Je ne sais pas si jamais nous deviendrons majeurs » (IV, 577). 
Dans cette voie, il envisage, avec Kant, la manière dont l'événement «Révolution française » a valeur de signe, non pas dans le caractère objectif du processus révolutionnaire lui-même, mais « dans la manière dont elle est accueillie tout alentour par des spectateurs qui n'y participent pas, mais qui la regardent, qui y assistent et qui, au mieux ou au pis, se laissent entraîner par elle» (IV, 684), ce que Kant appelle «la sympathie d'aspiration qui frise à l'enthousiasme ». Se sachant sous le regard du lecteur/spectateur de lui-même écrivant ses livres, il considère que l'actualité en devenir de son œuvre dépend de lecteurs/spectateurs qui construisent l'espace public à l'aide de signes liés à leur manière de remémorer l'événement, à leur façon de poser une perspective ouvrant à la compréhension de mon actualité.

Qu'en ressort-il à ce point ultime de l'œuvre de Foucault dans sa relation à lui-même ? Un moi actuel, moi-même au présent, en appui sur l'auteur anonyme, et au plus près du lecteur/spectateur du vécu, de l'action, du mouvement, y compris au sein même du processus constituant une œuvre. Foucault parle ici d' «éthique du souci de soi comme pratique de la liberté » en référence à «l'exercice de soi par soi par lequel on essaie de s'élaborer, de se transformer et d'accéder à un certain mode d'être » (IV, 709). Une fois écarté la construction d'un récit de soi à l'horizon d'un auteur consciemment défini par son œuvre, il s'en tient, si l'on peut dire, à l'exercice de soi par soi, à un récit de soi pris dans un perpétuel décalage, une sorte de jeu de langage. Ainsi les réflexions de ses ouvrages et articles oscillent en permanence entre ce que le titre en dit, et le problème autre posé par l'analyse en fin de parcours. Foucault ne se veut donc pas, à proprement parler, un auteur «effectif» par le fait de marquer sa présence dans l'histoire « réelle » d'aujourd'hui (IV, 704). Saisi par un « labeur infini », moi-même n'existe que dans le processus qui en concrétise son actualité aux yeux de ses lecteurs. La postérité intellectuelle de Michel Foucault, marquée par l'énorme succès international qu'on lui connait, lui donne raison $a$ posteriori.

\section{Bibliographie}

AMOSSY R., 2010, La présentation de soi. Ethos et identité verbale, Paris, PUF.

ATLAN C. \& LUCIANI I. (eds), 2014, Récits de soi, présence au monde: jugements et engagements (Europe, Afrique, XVIème -XXIème siècle), Aix-enProvence, PUP.

BARDET J.-P., ARNOUL E. \& RUGGIU F.-J. (eds), 2010, Les écrits du for privé en Europe du Moyen-Âge à l'époque contemporaine, Bordeaux, PUB.

BUTLER J., 2007, Le récit de soi, Paris, PUF.

FOUCAULT M., 1963, Raymond Roussel, Paris, Gallimard.

FOUCAULT M., 1971, L'ordre du discours, Paris, Gallimard. 
FOUCAULT M., 1972, Histoire de la folie à l'âge classique, Paris, Gallimard.

FOUCAULT M., 1994, Dits et Écrits, vol. I-IV, Paris, Gallimard.

FOUCAULT, 2008, Le gouvernement de soi et des autres, Paris, Gallimard.

Foucault, Cahiers de l'Herne, sous la dir. de ARTIERES P., BERT J.-F., GROS F. \& REVEL J., 2010, Paris, Éditions de l'Herne.

GUILHAUMOU J., 2006, Discours et événement. L'histoire langagière des concepts, Besançon, Presses Universitaires de Franche-Comté.

GUILHAUMOU J., 2013, «L'engagement d'un historien du discours : trajet et perspectives », Argumentation et analyse du discours, 11, Analyses du discours et engagement du chercheur sous la dir. de KOREN R., http://aad.revues.org/1599.

GUILHAUMOU J., 2014, «Michel Foucault et l'histoire généalogique », in LUCIANI I \& PIETRI V. (eds), Généalogies, Pouvoir, Histoire, Aix-enProvence, PUP.

HUSSERL E., 1976, La crise des sciences européennes et la phénoménologie transcendantale, Paris, Gallimard.

HUSSERL E., 1992, Méditations cartésiennes. Introduction à la phénoménologie, Paris, Vrin.

LUCIANI I \& PIETRI V. (eds), 2012, Écriture, récit, trouble(s) de soi. Perspectives historiques (France XVIème-XXème siècle), Aix-en-Provence, PUP.

PLATRINIERI L., 2012, L'expérience du concept. Michel Foucault entre épistémologie et histoire, Paris, Publications de la Sorbonne.

RIBARD D., 2003, Raconter, Vivre, Penser. Histoire de philosophes 1650-1766, Paris, Éditions Vrin-EHESS.

SAUSSURE F. de, 2002, Écrits de linguistique générale, Paris, Gallimard.

VIOULAC J., 2005, «De Nietzsche à Husserl. La phénoménologie comme accomplissement systématique du projet philosophique européen », Les Études philosophiques, 73, 203-227.

Résumé : La part du «récit de soi » dans les écrits de Michel Foucault est particulièrement difficile à cerner, dans le mesure où ne disposons pas, du moins dans son œuvre publiée, d'un texte autobiographique spécifique. Nous avons donc fait le choix du corpus de ses Dits et écrits où l'on trouve de nombreux entretiens, avec leur part de témoignage oral sur son parcours. Nous essayons de montrer que Foucault laisse l'écriture linéaire de son moi dans l'ombre, tout en déployant un moi dissocié, superposé à lui-même, volontairement lacunaire, et toujours ouvert sans pour autant déployer une écriture secrète, bien au contraire. Notre objectif est alors de poser quelques pistes sur ce qu'il en est de «soi-même » par la médiation de références littéraires et linguistiques dans l'espace de visibilité qu'il constitue sur la propre vérité de son «moi actuel », c'est-à-dire sur «moi-même », d'une période à l'autre de son trajet intellectuel. 
5 Mots-clés : récit de soi, réflexivité linguistique, archéologie du discours, généalogie historique, régime de vérité. 